\title{
The Shing-Tung Yau Center at Chiao Tung University
}

\author{
by Song-Sun Lin
}

Department of Mathematics, National Chiao Tung University, Hsinchu

The Shing-Tung Yau Center was founded on April 1, 2012 on behalf of Shing-Tung Yau, Professor of Mathematics at Harvard and awards winner for the Fields Medal (1982), the Crafoord Prize (1994), the National Medal of Science, U.S.A. (1997), and the Wolf Prize (2010). He acts as the Director, providing guidelines and directions for the Shing-Tung Yau Center.

The Shing-Tung Yau Center is committed to honor

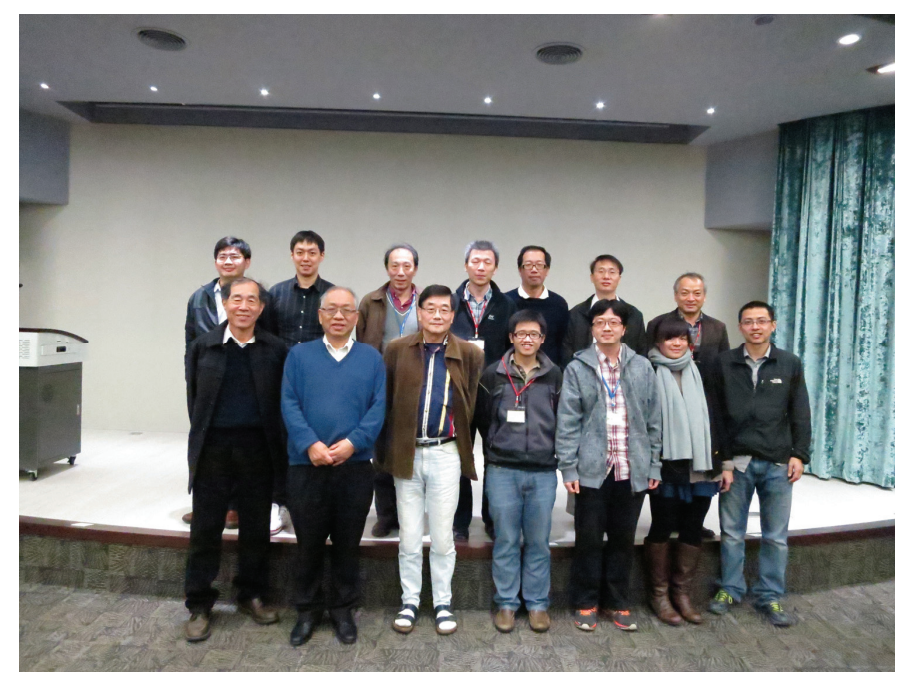

First row from left to right: Song-Sun Lin, Shing-Tung Yau and Wen-Wei Lin

Professor's Yau's contribution in Mathematics and Physics in order to promote the development of mathematics, science, technology, education, and culture.

The Shing-Tung Yau Center has around 20 affiliated faculty members drawn from the Departments of Applied Mathematics, the Institute of Statistics, the Electrophysics Department, the Computer Science Department, and the Institute of Photonics. Currently, Song-sun Lin, Lifetime National Chair Professor is the Executive Officer for the Shing-Tung Yau Center. There are 10 full-time research fellows at the Shing-Tung Yau Center, including 6 postdoctoral fellows and 4 research assistants.

\section{Main research areas of the Shing-Tung Yau Center}

- 3-D Imaging Group, led by Professor Wen-wei Lin in Applied Mathematics Department

- Medical Imaging Group, led by Dean Hong Xing Lu at College of Science

- Number Theory Group, led by Professor Yi Fang Yang in Applied Mathematics Department

- String Group, led by Professor Jen-chi Lee in Electrophysics Department

- Computational Complexity Group, led by Prof. Ker-I Ko in Computer Science Department

\section{Activity Highlights at the Shing-Tung Yau Center}

March 7, 2012 Invited Professor Shing-Tung Yau from Harvard University to give a talk on the topic of "Geometry Appreciation"

July 6-7, 2012 Invited Academicians of Academia Sinica and Professors Shing-Tung Yau from Harvard University,Wing Hung Wong from Stanford University, Ching-Li Chai from University of Pennsylvania, Jin-Chuan Duan from National University of Singapore, and Horng-Tzer Yau from Harvard University to provide a series of talks with a subject of "Meeting the Masters"

Dec. 10-11, 2012 International Conference on "3D Imaging and Computing" was successfully conducted. Over 200+ participants attended the conference. 